\title{
Diagnostic Performance of Routine Brain MRI Sequences for Dural Venous Sinus Thrombosis
}

\author{
(DD. Patel, (D) Machnowska, (D) S. Symons, (DR. Yeung, (D)A.J. Fox, (DR.I. Aviv, and DP. Jabehdar Maralani
}

\begin{abstract}
BACKGROUND AND PURPOSE: Signs suggestive of unexpected dural venous sinus thrombosis are detectable on routine MR imaging studies without MRV. We assessed performance characteristics and interrater reliability of routine MR imaging for the diagnosis of dural venous sinus thrombosis, focusing on the superior sagittal, transverse, and sigmoid sinuses.
\end{abstract}

MATERIALS AND METHODS: This case series included 350 patients with MRIs performed with contrast-enhanced MRV and 79 patients with routine MRIs performed within 48 hours of a CTV from 2008 to 2014 (total, $n=429$ ). Routine MR images were separated from the contrast-enhanced MRVs and CTVs. Three neuroradiologists, blinded to clinical data, independently reviewed the MRIs for signs of dural venous sinus thrombosis, including high signal on sagittal T1, loss of flow void on axial T2, high signal on FLAIR, high signal on DWI, increased susceptibility effects on $\mathrm{T} 2{ }^{*}$-weighted gradient recalled-echo imaging, and filling defects on axial contrast-enhanced spin-echo TIWI and/or volumetric gradient-echo TIWI. Two neuroradiologists independently reviewed contrast-enhanced MRVs and CTVs to determine the consensus gold standard. Interrater reliability was calculated by using the $\kappa$ coefficient.

RESULTS: Contrast-enhanced MRV and CTV confirmed that dural venous sinus thrombosis was present in 72 of 429 cases (16.8\%). The combination of routine MR sequences had an overall sensitivity of $79.2 \%$, specificity of $89.9 \%$, and moderate interrater reliability $(\kappa=0.50)$. The 3 readers did not have similar performance characteristics. $69.4 \%$ of positive cases had clinical suspicion of dural venous sinus thrombosis indicated on imaging requisition.

CONCLUSIONS: Routine MR images can suggest dural venous sinus thrombosis with high specificity in high-risk patients, even in cases without clinical suspicion.

ABBREVIATIONS: CE-3D-TIWI = volumetric contrast-enhanced GRE TI-weighted imaging; CEMRV = contrast-enhanced MRV; CE-SE-TIWI = contrast-enhanced spin-echo TT-weighted imaging; DVST $=$ dural venous sinus thrombosis; GRE $=T 2^{*}$-weighted gradient recalled-echo imaging

D ural venous sinus thrombosis (DVST) is a condition that ranges from being undiagnosed to leading to serious morbidity and mortality, including venous infarction and intracranial hemorrhage. ${ }^{1}$ DVST has a highly variable clinical presentation, from asymptomatic to acute or subacute headaches, signs or symptoms of increased intracranial pressure, focal neurologic deficits, or seizures. ${ }^{2,3}$ The diagnosis is important to consider even in younger patients, with risk factors such as genetic and acquired

Received February 1, 2016; accepted after revision April 25.

From the Department of Medical Imaging, University of Toronto, Sunnybrook Health Sciences Centre, Toronto, Ontario, Canada.

Paper previously presented at: Annual Meeting of the American Society of Neuroradiology and the Foundation of the ASNR Symposium, April 25-30, 2016; Chicago, Illinois.

Please address correspondence to Pejman Jabehdar Maralani, MD, FRCPC, Department of Medical Imaging, University of Toronto, Sunnybrook Health Sciences Centre, AG 270C, 2075 Bayview Ave, Toronto, ON, M4N 3M5; e-mail: Pejman.Maralani@sunnybrook.ca

http://dx.doi.org/10.3174/ajnr.A4843 prothrombotic states, trauma, and infections or inflammatory conditions. $^{1,2}$

Although early and accurate diagnosis is important for initiation of treatment and prevention of serious complications, the median diagnostic delay from symptom onset is 7 days. ${ }^{1-4}$ When DVST is suspected, the current criterion standard for diagnosis is either contrast-enhanced MRV (CEMRV) or CTV. ${ }^{5}$ However, the imaging signs of unexpected DVST are seen during interpretation of routine MR imaging studies performed without dedicated CEMRV, concurrent CTV, or relevant clinical history. Typical findings might include visualization of intraluminal thrombus or a filling defect and lack of flow, or flow voids. ${ }^{2}$ Signs suggestive of DVST on standard brain MR imaging are reported in the literature with highly variable diagnostic accuracy and can be difficult to interpret in daily practice. ${ }^{2,6-8}$ Understanding the diagnostic performance of routine MR imaging for the evaluation of DVST is especially important when DVST is not clinically suspected or CT or MR venography has not been ordered. A false-positive inter- 


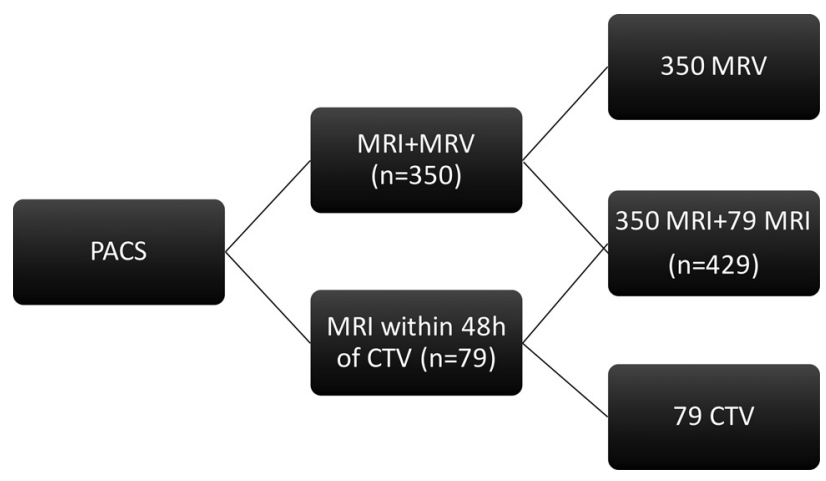

FIG 1. Patient selection and image separation.

pretation of DVST based on routine MR imaging will lead to unnecessary patient anxiety, require further imaging and associated health care costs, and increase patient risk from exposure to contrast media or radiation from CTV. False-negative interpretations can lead to devastating complications such as intracranial venous hypertension, hemorrhage, and venous infarction. The aim of this study was to assess the performance characteristics and interrater reliability of signs of DVST, not including cortical or deep vein thrombosis, on routine brain MR imaging, including T1, T2, T2*-weighted gradient recalled-echo imaging (GRE), FLAIR, DWI, and postcontrast T1 sequences in comparison with CEMRV and CTV as the reference standard.

\section{MATERIALS AND METHODS \\ Patient Selection}

This case series study was approved by the institutional review board with a waiver of informed consent. The PACS was searched for patients who had both MR imaging and CEMRV or MR imaging and CTV from April 2008 to June 2014. The study included 350 MRIs performed with CEMRV and 79 MRI studies performed within 48 hours of a CTV for a total of 429 patients (Fig 1). Patients with prior surgical intervention that altered the anatomy of the dural venous sinuses were excluded because the dural sinuses cannot be assessed without a dedicated venogram study. No patients received anticoagulation between MR imaging and CTV. The patients were numbered in the order of their imaging study retrieval. Demographics and clinical information, including underlying conditions and indications for imaging, were recorded in a separate data base.

\section{Imaging Protocol}

MR imaging was performed by using 1.5T TwinSpeed Excite (GE Healthcare, Milwaukee, Wisconsin) and 3T Achieva TX (Philips Healthcare, Best, the Netherlands) scanners using a standard 8-channel head coil.

The MR imaging sequences of interest and parameters (TR/ TE/flip angle) for 1.5T were the following: sagittal T1 (2163 ms/ $\left.25.5 \mathrm{~ms} / 90^{\circ}\right)$, axial T2 $\left(1067 \mathrm{~ms} / 35 \mathrm{~ms} / 20^{\circ}\right)$, axial T2 FLAIR $(8600$ $\left.\mathrm{ms} / 114.3 \mathrm{~ms} / 90^{\circ}\right)$, axial GRE $\left(1200 \mathrm{~ms} / 35 \mathrm{~ms} / 20^{\circ}\right)$, axial DWI (7000 ms/71.4 ms $\left./ 90^{\circ}\right)$, axial contrast-enhanced spin-echo $\mathrm{T} 1$ (CE-SE-T1WI) $\left(350 \mathrm{~ms} / 20 \mathrm{~ms} / 0^{\circ}\right)$, and volumetric contrast-enhanced GRE T1 (CE-3D-T1WI) $\left(8.6 \mathrm{~ms} / 4.2 \mathrm{~ms} / 20^{\circ}\right)$. The MR imaging sequences of interest and parameters (TR/TE/flip angle) for 3T were the following: T1 $\left(9.5 \mathrm{~ms} / 2.3 \mathrm{~ms} / 8^{\circ}\right)$, T2 (3793 ms/120 $\left.\mathrm{ms} / 90^{\circ}\right)$, FLAIR $\left(9000 \mathrm{~ms} / 125 \mathrm{~ms} / 90^{\circ}\right), \operatorname{GRE}\left(812 \mathrm{~ms} / 16 \mathrm{~ms} / 18^{\circ}\right)$, DWI (3339 ms $\left./ 45.8 \mathrm{~ms} / 90^{\circ}\right)$, SWI $\left(15.9 \mathrm{~ms} / 22.5 \mathrm{~ms} / 15^{\circ}\right)$, CE-SET1WI $\left(553.7 \mathrm{~ms} / 13 \mathrm{~ms} / 90^{\circ}\right)$, and CE-3D-T1WI $(9.5 \mathrm{~ms} / 2.3 \mathrm{~ms} /$ $8^{\circ}$ ). CEMRV was performed with the following parameters (TR/ TE/flip angle) for $1.5 \mathrm{~T}$ and 3T: $4.2 \mathrm{~ms} / 1.4 \mathrm{~ms} / 25^{\circ}$ and $3.9 \mathrm{~ms} / 1.5$ $\mathrm{ms} / 30^{\circ}$. The patients were injected with $0.1 \mathrm{mmol} / \mathrm{kg}$ of gadobutrol (Gadovist; Bayer Schering Pharma, Berlin, Germany), maximum of $10 \mathrm{~mL}$, by using a bolus-tracking technique and a power injector (Spectris MR injector; MedRad, Indianola, Pennsylvania) at a rate of $1.5 \mathrm{~mL} / \mathrm{s}$.

CTV was performed by using a LightSpeed 64-section scanner (GE Healthcare) as a dedicated protocol from C3 to the vertex, 0.625-mm helical. Iodixanol (Visipaque 320; GE Healthcare, Piscataway, New Jersey) was administered intravenously at an injection rate of 3-4 mL/s by using a bolus-tracking technique. Both CTV and CEMRV images had coronal, sagittal, and axial maximum-intensity-projection reformats. CT angiograms with good opacification of the dural venous sinuses were not included in the study because we wanted to use only dedicated CTV or CEMRV as our gold standard.

\section{Image Analysis}

Images were anonymized and downloaded to an off-line workstation and were separated into 3 datasets: first, MR images with sequences of interest including T1, T2, GRE, FLAIR, DWI, SWI, CE-SE-T1WI, and CE-3D-T1WI without any vascular imaging; second, CTV source images with MIPs; and third, CEMRV source images with MIPs (Fig 1).

Of the 429 cases, the routine MR imaging dataset contained 418 cases with T1, 71 with T2, 420 with GRE, 417 with FLAIR, 421 with DWI, 139 with SWI, 241 with CE-SE-T1WI, and 199 with CE-3D-T1WI. Of the 72 cases positive for DVST by CEMRV or CTV, there were 71 cases with T1, 8 with T2, 70 with GRE, 69 with FLAIR, 71 with DWI, 15 with SWI, 44 with CE-SE-T1WI, and 23 with CE-3D-T1WI. The smaller sample sizes of T2, CE-SE-T1WI, and CE-3D-T1WI occurred because axial T2 was eliminated from the routine brain MRI and MRV at our institution in mid-2008 and CE-SE-T1WI was replaced by CE-3D-T1WI in 2010.

Signs of DVST investigated included high signal on sagittal T1, loss of flow void on axial T2, loss of flow void or hyperintense signal on FLAIR, increased susceptibility effects on axial GRE and SWI, high signal on axial DWI, and the presence of filling defects on axial CE-SE-T1WI and/or on CE-3D-T1WI. In each case, evaluation of all sequences of interest was performed at the same time to reflect usual clinical practice.

Three neuroradiologists, blinded to patient information and reports, independently reviewed the MR imaging sequence of interest for signs of DVST. In each case, the readers indicated the presence or absence of DVST as their overall impression. They also stated which individual sequences demonstrated findings of DVST. Readers had access to each of the MR imaging sequences of interest at the same time. Readers evaluated the superior sagittal sinus, right and left transverse sinuses, and right and left sigmoid sinuses.

Two neuroradiologists, blinded to patient information, reports, and the results from the sequence-of-interest analysis, independently reviewed CEMRV and CTV datasets to determine 
the consensus reference standard. These neuroradiologists did not review the standard MR images from the CEMRV as in clinical practice. Disagreement was resolved by consensus after review by a third neuroradiologist. The cases were reviewed in multiple 1-hour sessions to reduce the influence of reader fatigue.

\section{Data Analysis}

Individual reader results were compared against the reference standard and used to determine reader sensitivity, specificity, and accuracy for the combination of routine MR imaging signs. The performance characteristics of individual readers were compared with the McNemar test and SPSS software, Version 20.0 (IBM, Armonk, New York). Although in clinical practice MR imaging studies are read by individual radiologists, for this study, the performance of routine MR imaging was calculated on the basis of the most votes of the 3 readers when the sequences of interest were combined. The cases were also analyzed by comparing results from the 1.5T-versus-3T MR imaging scanners. Positive predictive and negative predictive values and accuracies of the consensus read were not reported because of the selective sample of this study and lack of prevalence data. Interrater reliability was calculated for each MR imaging sequence individually and combined by using $\kappa$ coefficients with SAS 9.4 (SAS Institute, Cary, North Carolina). The agreement was considered slight if $\kappa$ values were $0-0.20$; fair if, 0.21-0.40; moderate if, 0.41-0.60; substantial if, 0.61-0.80; and almost perfect if, 0.81-1. ${ }^{9}$ Results were expressed as mean \pm SD. $P<.05$ was considered significant.

Table 1: Patient characteristics

\begin{tabular}{lccc}
\hline & DVST $(\boldsymbol{n}=\mathbf{7 2})$ & Non-DVST $(\boldsymbol{n}=\mathbf{3 5 7})$ & Total $(\boldsymbol{n}=\mathbf{4 2 9})$ \\
\hline Age (yr) (mean) & $51.4 \pm 16.8$ & $45.8 \pm 17.0$ & $46.7 \pm 17.1$ \\
Female (No.) (\%) & $45(62.5)$ & $239(66.9)$ & $284(66.2)$ \\
3T MRI (No.) (\%) & $16(22.2)$ & $130(36.4)$ & $146(34.0)$ \\
\hline
\end{tabular}

\section{RESULTS}

\section{Clinical Findings}

There were 145 men and 284 women with a mean age of $46.7 \pm$ 17.1 years (Table 1). Women composed more than one-half of the sample volume (62.5\%) and cases positive for DVST (66.2\%). Two-thirds of the cases were performed on a 1.5T scanner (Table 1 ).

CEMRV and CTV confirmed that DVST was present in 72/429 cases $(16.8 \%)$. The mean age of patients with DVST was $51.4 \pm$ 16.8 years.

The most common underlying predisposition for DVST was primary brain neoplasm or brain metastasis (19.4\%), followed by traumatic brain injury (13.9\%) and intracranial hemorrhage (subdural, subarachnoid, or intraparenchymal hemorrhage) (12.5\%) (Fig 2). Only 1 patient demonstrated an underlying coagulation disorder (antiphospholipid antibody syndrome). Infectious or inflammatory factors included systemic lupus erythematosus, herpes simplex encephalitis, vasculitis, and abscess/ osteomyelitis at the skull base (6.94\%). Two patients in the intensive care unit were positive for DVST without a clear etiology (Fig 2).

The most commonly stated clinical indication for imaging was suspicion of DVST (Fig 3). For the cases positive for DVST, 69.4\% had suspicion of DVST indicated, so $30.6 \%$ of positive cases were clinically unexpected DVST. Of the total 207 cases imaged with the explicit indication of "rule out DVST," 50 (24.2\%) were positive for it.

\section{Performance Characteristics}

When we compared the performance characteristics of the 3 readers, reader 1 had significantly higher sensitivity than reader 2 , and reader 2 had significantly higher specificity and accuracy than both readers 1 and 3 (Table 2). The most accurate reader (87.7\%) had higher specificity (91.4\%) at the expense of lower sensitivity $(70.8 \%)$.

Routine MR imaging had an overall sensitivity of $79.2 \%$ and specificity of $89.9 \%$ based on the most votes of the 3 readers when

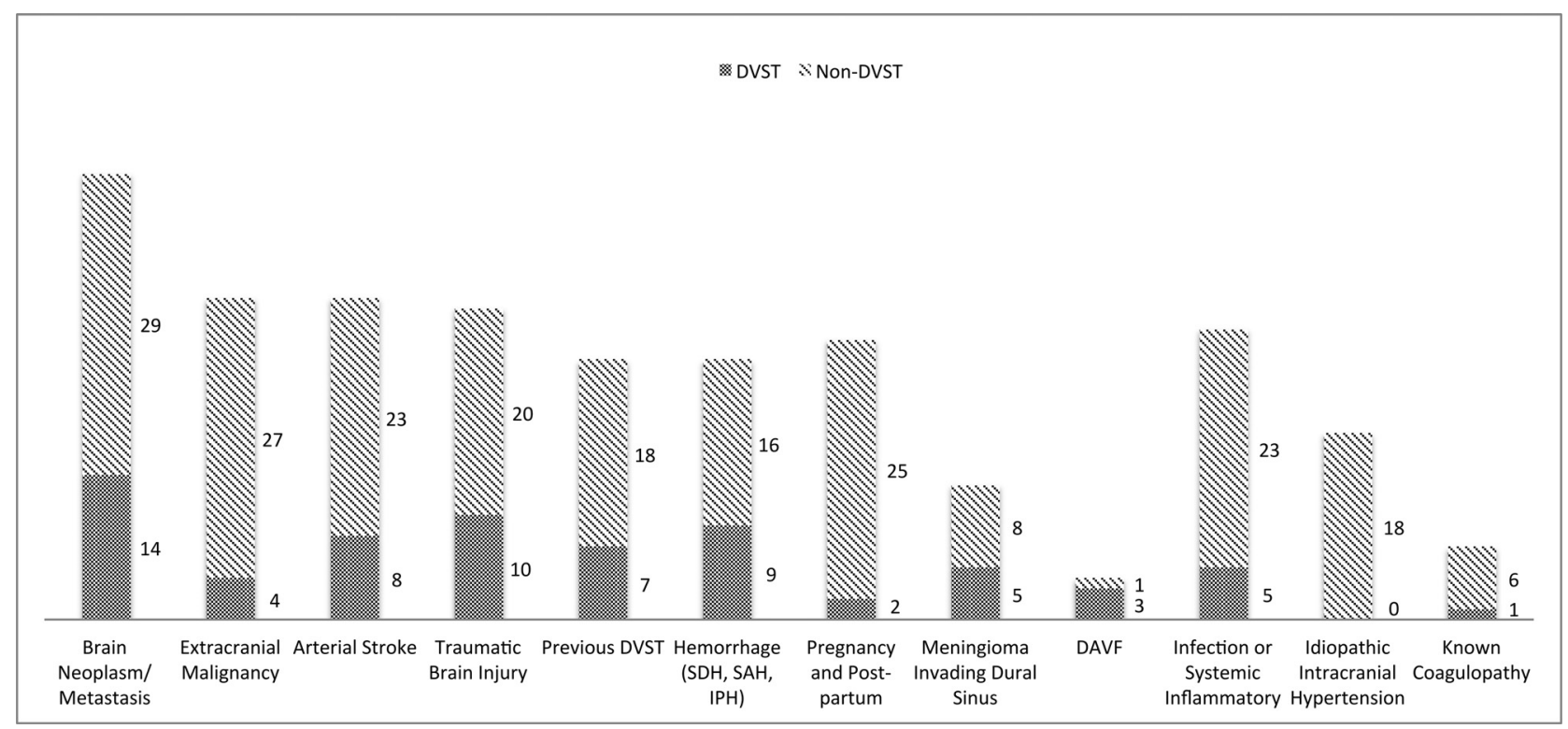

FIG 2. Etiology or underlying condition based on electronic medical records. SDH indicates subdural hemorrhage; IPH, intraparenchymal hemorrhage; DAVF, dural arteriovenous fistula. 


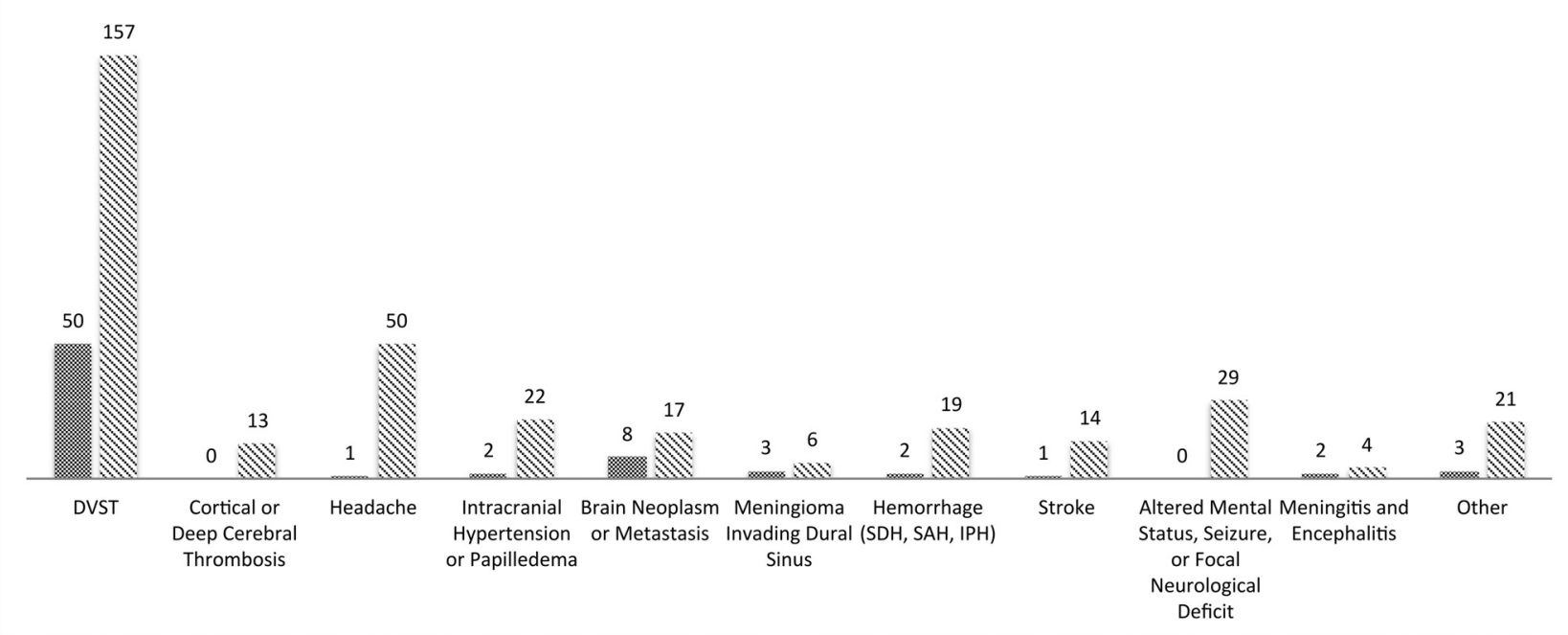

FIG 3. Indications for imaging based on the imaging requisition form. SDH indicates subdural hemorrhage; IPH, intraparenchymal hemorrhage.

Table 2: Performance of routine MRI sequences compared with CEMRV or CTV for each reader

\begin{tabular}{lccc} 
& Sensitivity (\%) & Specificity (\%) & Accuracy (\%) \\
\hline Reader 1 & $84.7^{\mathrm{a}}$ & 82.1 & 82.5 \\
Reader 2 & 70.8 & $91.4^{\mathrm{b}}$ & $87.7^{\mathrm{b}}$ \\
Reader 3 & 72.2 & 84.3 & 82.3 \\
\hline
\end{tabular}

a Statistically significant compared with reader $2(P<.05)$.

${ }^{\mathrm{b}}$ Statistically significant compared with readers 1 and $3(P<.05)$

Table 3: Performance of MRI for the detection of DVST compared with CEMRV or CTV based on the most votes Sensitivity (\%) Specificity (\%)

\begin{tabular}{lll}
\hline MRI total $(n=429)$ & 79.2 & 89.9 \\
MRI total for 1.5T $(n=283)$ & $85.7^{\mathrm{a}}$ & 87.2 \\
MRI total for 3T $(n=146)$ & $56.3^{\mathrm{b}}$ & $94.6^{\mathrm{c}}$ \\
\hline
\end{tabular}

a Statistically significant compared with MRI Total $(P<.05)$.

${ }^{b}$ Statistically significant compared with MRI Total and 1.5T $(P<.05)$

'Statistically significant compared with 1.5T $(P<.05)$.

the sequences of interest were combined (Table 3). The 3T scanner had significantly lower sensitivity compared with both $1.5 \mathrm{~T}$ and total MR imaging and significantly higher specificity than 1.5T (Table 3). MR imaging signs of DVST are shown in Fig 4. SWI was not included in the analysis because it was not diagnostic due to susceptibility artifacts from the contents of the dural venous sinuses and the adjacent skull, and no interpretation could be made. There were no cases positive for DVST based on SWI. All cases with high signal on DWI $(n=14)$ were associated with abnormal signal changes on T1 and/or FLAIR. Of the 31 cases true-positive for DVST on GRE, 12 were false-negative on T1; 2 cases, on T2; 20 cases, on DWI; 5 cases, on FLAIR; 8 cases, on CE-SE-T1WI; and no cases, on CE-3D-T1WI. GRE had true-positive findings in 6 cases that had false-negative findings on all the other sequences other than CE-3D-T1WI.

The interrater reliability with $\kappa$ statistics demonstrated fair agreement for the non-contrast-enhanced sequences and moderate agreement for the contrast-enhanced sequences (Table 4).

\section{DISCUSSION}

DVST is a relatively rare condition, and previous studies of DVST have had small sample sizes. This is a large study of 429 cases investigating the diagnostic accuracy of routine MR imaging for DVST. Each case was reviewed by 3 neuroradiologists. Due to the nonspecific nature of the clinical presentations, which may be asymptomatic, the radiologist reviewing standard brain MR imaging may be the first to consider the diagnosis of DVST. This study demonstrates that abnormal dural venous sinus signal on 7 common routine MR sequences can suggest the diagnosis of DVST with high specificity.

While data from Coutinho et $\mathrm{al}^{10}$ and the International Study on Cerebral Vein and Dural Sinus Thrombosis demonstrated a female/male ratio of 3:1, with a median age of 34 years compared with 42 years, respectively, in our study, the female/male ratio for cases positive for DVST was less than expected (1.7:1) and patients diagnosed with DVST were older than expected $(51.4 \pm$ 16.8 years). Furthermore, whereas pregnancy, postpartum status, and oral contraceptives are among the most common underlying conditions in cases of DVST, ${ }^{3,11}$ this was not true in our study. Neoplasia was a much more common etiologic factor compared with previous studies such as that of Lafitte et al, ${ }^{11}$ in which neoplasia was identified in only 2 of 20 patients with positive findings. These results are most likely due to the selective group of patients presenting to our institution and the selection bias of our study. The patient population was from a large tertiary trauma, stroke, and cancer center, which helps explain the different demographics compared with previous studies.

Although DVST is not a common condition, there were 72 cases positive for it among 429 studied venograms (16.8\%) at our tertiary care academic health center. These did not include any CTV studies that were not associated with MR imaging evaluation or any other patients who went undiagnosed. The prevalence of cerebral venous thrombosis has been estimated to be 5/1,000,000 in the general population, and a postmortem series of 182 subjects found an incidence of 9.3\%. ${ }^{12,13}$ In this study, cases were selected 


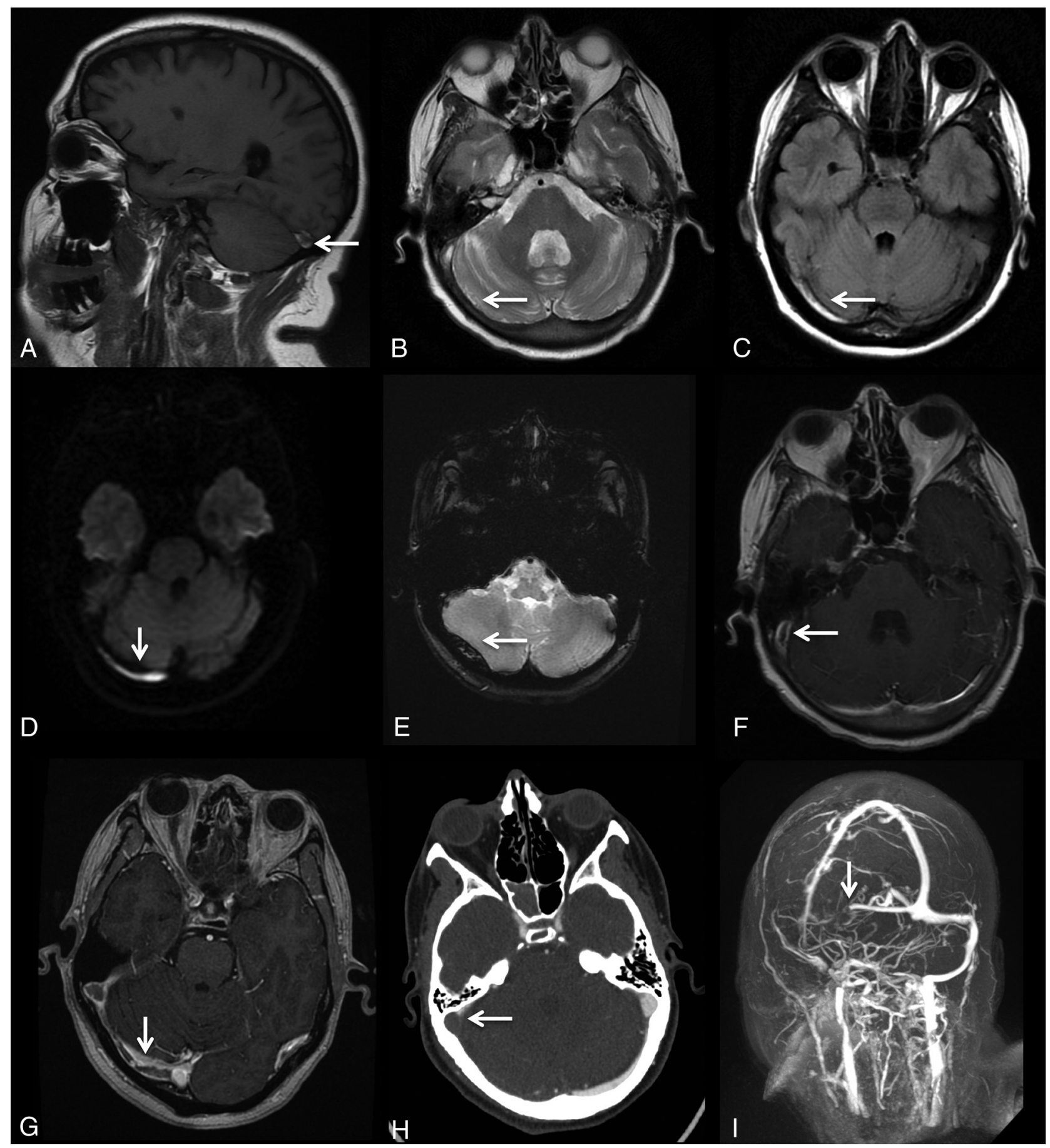

FIG 4. MR imaging signs of dural venous sinus thrombosis from multiple cases. $A$, Hyperintense signal in the right transverse sinus on sagittal TI. $B$, Loss of flow void in the right transverse sinus on axial T2. C, Hyperintense signal in the right transverse sinus on FLAIR. D, Hyperintense signal in the right transverse sinus on DWI. E, Blooming artifacts in the right transverse sinus on GRE. F, Filling defect in the right transverse/sigmoid sinus on CE-SE-TIWI. G, Filling defect in the right transverse/sigmoid sinus on CE-3D-TIWI. H, Filling defect in the right transverse/sigmoid sinus on CTV. I, Filling defect in the right transverse/sigmoid sinus on MIP-CEMRV.

from patients who had CTV or CEMRV, which puts them at a substantially higher pretest probability of having DVST than the general population, and this does not represent the prevalence of the disease. Therefore, positive predictive value, negative predictive value, and accuracy were not reported. While this omission limits the external validity of the study, the performance characteristics are relevant to those in similar populations in tertiary care centers.
Tang et $\mathrm{al}^{14}$ found that DVST was indicated on the imaging requisitions in only $33 \%$ of MR imaging studies positive for thrombosis, ${ }^{6}$ compared with our study in which $69.4 \%$ of cases positive for DVST had "suspected DVST" on their imaging requisitions. Some other indications for venography such as headache, intracranial hypertension, and meningioma invading the dural venous sinuses may be the cause or the effect of DVST. Thus, $30.6 \%$ of cases positive for DVST were found in patients 
Table 4: Interrater reliability for each MRI sequence individually and combined using the $\kappa$ coefficient

\begin{tabular}{lll}
\hline & $\boldsymbol{\kappa}$ & Agreement $^{\mathbf{a}}$ \\
\hline Sagittal TI $(n=418)$ & 0.28 & Fair \\
Axial T2 $(n=71)$ & 0.34 & Fair \\
Axial GRE $(n=420)$ & 0.40 & Fair \\
Axial FLAIR $(n=417)$ & 0.34 & Fair \\
Axial DWI $(n=421)$ & 0.33 & Fair \\
Axial CE-SE-TIWI $(n=241)$ & 0.42 & Moderate \\
Axial CE-3D-TIWI $(n=199)$ & 0.41 & Moderate \\
MRI total $(n=429)$ & 0.50 & Moderate \\
\hline
\end{tabular}

agreement was considered slight if $\kappa$ values were $0-0.20$; fair if, 0.21-0.40; moderate if, 0.41-0.60; substantial if, 0.61-0.80; and almost perfect if, 0.81-1.

without clinical suspicion of it, highlighting the importance of routine MR imaging signs of DVST in unexpected situations.

The overall sensitivity of MR imaging (79.2\%) was slightly lower than that in previously reported studies. Lafitte et $\mathrm{al}^{11}$ reported that in 20 patients with clinically suspected DVST confirmed by digital subtraction angiography, MR imaging that included spin-echo T1- and T2-weighted imaging was 90\% sensitive in diagnosing DVST, and $>80 \%$ sensitivity was found in most studies. This finding can be explained, in part, by the high sample volume in our study, which is likely closer to the real performance of the sequence of interest in the diagnosis of DVST in samples from a tertiary care center.

The performance characteristics in this study are in keeping with the findings of Saindane et al, ${ }^{7}$ who demonstrated that filling defects on CE-3D-T1WI had a sensitivity of $67 \%$ and a specificity of $100 \%$ for DVST compared with MRV. Sari et $\mathrm{al}^{8}$ demonstrated that immediate CE-3D-T1WI had a sensitivity of $92.5 \%$, specificity of $100 \%$, and accuracy of $98.3 \%$ compared with their reference standard. They proposed that delayed acquisition of CE-3D-T1WI after contrast injection can lead to contrast enhancement of the thrombus, which lowers the sensitivity of the sequence. ${ }^{8}$

The reported sensitivity for hyperintense signal on DWI for intraluminal thrombus has been highly variable in the literature, ranging from $4 \%$ to $40 \% .^{2}$ Our results corroborate the study by Favrole et al, ${ }^{15}$ which noted that a high DWI signal is always found with concomitant signal changes on T1 and/or FLAIR sequences; thus, it may not provide much diagnostic value.

GRE identified cases that were false-negative for DVST on all of the other sequences other than CE-3D-T1WI, providing additional diagnostic value as part of a standard brain MR imaging. These results are consistent with the findings of Ihn et al, ${ }^{16}$ who demonstrated that GRE was the only sequence that was positive for DVST in all 11 DVST cases in a study that included T1, T2, FLAIR, and CE-SE-T1WI. Altinkaya et $\mathrm{al}^{17}$ have shown that GRE has high diagnostic performance for acute and subacute superior sagittal sinus and deep and cortical vein thrombosis, but not for transverse and sigmoid sinus thrombosis due to susceptibility artifacts from the skull base.

Multiple reasons have been proposed for the low sensitivity of MR imaging sequences for DVST. Hinman and Provenzale ${ }^{18}$ reported that though in most cases, the diagnosis of DVST can be made on routine MR imaging on the basis of the loss of a normal flow void with abnormal signal intensity within the dural venous sinus, during the first 5 days after formation, an acute thrombus can mimic a normal flow void on spin-echo T2-weighted imaging and may be isointense with gray matter on spin-echo T1-weighted imaging. ${ }^{16,19}$ In this case, the hypointense signal on T2 can be mistaken for patency. In the subacute stage, the thrombus becomes progressively hyperintense on both spin-echo T1- and T2weighted imaging. ${ }^{11}$ Enhancement of a chronic thrombus can resemble normal flow. ${ }^{7,20,21}$ In the chronic stage, contrast-enhanced T1-weighted images of DVST can enhance to resemble a patent sinus due to capillary channels or partial recanalization. ${ }^{6,7}$ Routine MR images need to be evaluated with caution to avoid false-negative interpretations.

In our study, the specificity for DVST was high; this finding indicates a low number of false-positive interpretations. Falsepositives can occur if there is slow blood flow due to sinus hypoplasia, which can create a high signal that resembles a thrombus. This pitfall can be avoided by using multiple sequences and planes of section. ${ }^{22}$ Filling defects caused by arachnoid granulations or other anatomic material in the sinuses may also resemble thrombus, but signal isointense to CSF and morphology can help differentiate them. ${ }^{6,23}$

In terms of interrater reliability, Ferro et $\mathrm{al}^{24}$ showed that with a sample size of 40 cases, interrater reliability for DVST varies from moderate to excellent, $\kappa$ range of $0.59-1$, when comparing pairs of raters. In this study, for the individual MR images, there was considerable variability because $\kappa$ statistics ranged from 0.28 to 0.42 . The interrater reliability for overall thrombosis diagnosis was only moderate at 0.50 , which reflects the difficulty in interpreting the signs suggestive of DVST. Individual radiologists each have their own propensity to make the diagnosis, which may explain why the performance characteristics of the 3 readers had significant differences.

\section{Limitations}

Although readers were blinded to clinical history and whether venography was completed, readers were focused on searching for DVST, and the sample was chosen on the basis of patients who had undergone CEMRV or CTV in a tertiary care center. These features substantially increased the pretest probability of DVST compared with a random sample and promoted selection bias. This high prevalence would falsely increase the accuracy of MR imaging for DVST among the 3 readers. The accuracy of the readers was only reported to demonstrate individual differences, but it is not generalizable to daily practice for the reasons described above. Readers had access to some or all of the MR images (T1, T2 and/or FLAIR, GRE, DWI, SWI, CE-SE-T1WI, and/or CE-3DT1WI), which would confound individual sequence test parameters. The neuroradiologists may have used secondary clues to the diagnosis other than those of interest such as hemorrhage, edema, or infarction to help them. The study included 18 patients with idiopathic intracranial hypertension, which is known to have abnormal transverse sinus morphology and flow; however, none of these patients were positive for DVST. In addition, the age of the thrombosis was not taken into account. The signal characteristics are dependent on the age of the thrombosis, and Idbaih et $\mathrm{al}^{25}$ have shown that the sensitivity of routine MR images changes as the thrombus evolves.

AJNR Am J Neuroradiol 37:2026-32 Nov 2016 www.ajnr.org 


\section{CONCLUSIONS}

Abnormal dural venous sinus signal on routine MR images can suggest DVST with high specificity and moderate interrater reliability in high-risk patients. In patients with primary or metastatic brain cancer, these signs need to be taken very seriously, and this is particularly important in patients in whom there is no clinical suspicion of DVST.

Disclosures: Richard I. Aviv—UNRELATED: Grants/Grants Pending: Canadian Institutes of Health Research*; Other: Biogen Foundation fellowship.* Pejman Jabehdar Maralani-UNRELATED: Grants/Grants Pending: Radiological Society of North America, ${ }^{*}$ Brain Tumor Foundation of Canada, ${ }^{*}$ Comments: Both grants are related to a brain tumor project and not related to the submitted work. *Money paid to the institution.

\section{REFERENCES}

1. Stam J. Thrombosis of the cerebral veins and sinuses. $N$ Engl J Med 2005; 352:1791-98 CrossRef Medline

2. Linn J, Brückmann H. Cerebral venous and dural sinus thrombosis: state-of-the-art imaging. Clin Neuroradiol 2010;20:25-37 CrossRef Medline

3. Ferro JM, Canhão P, Stam J, et al; ISCVT Investigators. Prognosis of cerebral vein and dural sinus thrombosis: results of the International Study on Cerebral Vein and Dural Sinus Thrombosis (ISCVT). Stroke 2004;35:664-70 Medline

4. Ferro JM, Canhão P, Stam J, et al; ISCVT Investigators. Delay in the diagnosis of cerebral vein and dural sinus thrombosis: influence on outcome. Stroke 2009;40:3133-38 CrossRef Medline

5. Khandelwal N1, Agarwal A, Kochhar R, et al. Comparison of CT venography with MR venography in cerebral sinovenous thrombosis. AJR Am J Roentgenol 2006;187:637-43 Medline

6. Provenzale JM, Kranz PG. Dural sinus thrombosis: sources of error in image interpretation. AJR Am J Roentgenol 2011;196:23-31 CrossRef Medline

7. Saindane AM, Mitchell BC, Kang J, et al. Performance of spin-echo and gradient-echo T1-weighted sequences for evaluation of dural venous sinus thrombosis and stenosis. AJR Am J Roentgenol 2013; 201:162-69 CrossRef Medline

8. Sari S, Verim S, Hamcan S, et al. MRI diagnosis of dural sinuscortical venous thrombosis: immediate post-contrast 3D GRE T1-weighted imaging versus unenhanced MR venography and conventional MR sequences. Clin Neurol Neurosurg 2015;134: 44-54 CrossRef Medline

9. Altman DG. Practical Statistics for Medical Research. London: Chapman and Hall; 1991

10. Coutinho JM, Ferro JM, Canhão $\mathrm{P}$, et al. Cerebral venous and sinus thrombosis in women. Stroke 2009;40:2356-61 CrossRef Medline

11. Lafitte F, Boukobza M, Guichard JP, et al. MRI and MRA for diagno- sis and follow-up of cerebral venous thrombosis (CVT). Clin Radiol 1997;52:672-79 CrossRef Medline

12. Towbin A. The syndrome of latent cerebral venous thrombosis: its frequency and relation to age and congestive heart failure. Stroke 1973;4:419-30 CrossRef Medline

13. Saposnik G, Barinagarrementeria F, Brown RD Jr, et al; American Heart Association Stroke Council and the Council on Epidemiology and Prevention. Diagnosis and management of cerebral venous thrombosis: a statement for healthcare professionals from the American Heart Association/American Stroke Association. Stroke 2011;42:1158-92 CrossRef Medline

14. Tang PH, Chai J, Chan $\mathrm{YH}$, et al. Superior sagittal sinus thrombosis: subtle signs on neuroimaging. Ann Acad Med Singapore 2008;37: 397-401 Medline

15. Favrole P, Guichard JP, Crassard I, et al. Diffusion-weighted imaging of intravascular clots in cerebral venous thrombosis. Stroke 2004; 35:99-103 Medline

16. Ihn YK, Jung WS, Hwang SS. The value of T2*-weighted gradientecho MRI for the diagnosis of cerebral venous sinus thrombosis. Clin Imaging 2013;37:446-450 CrossRef Medline

17. Altinkaya N, Demir S, Alkan O, et al. Diagnostic value of T2* weighted gradient-echo MRI for segmental evaluation in cerebral venous sinus thrombosis. Clin Imaging 2015;39:15-19 CrossRef Medline

18. Hinman JM, Provenzale JM. Hypointense thrombus on T2weighted MR imaging: a potential pitfall in the diagnosis of dural sinus thrombosis. Eur J Radiol 2002;41:147-52 CrossRef Medline

19. Dormont D, Anxionnat R, Evrard, et al. MRI in cerebral venous thrombosis. J Neuroradiol 1994;21:81-99 Medline

20. Dormont D, Sag K, Biondi A, et al. Gadolinium-enhanced MR of chronic dural sinus thrombosis. AJNR Am J Neuroradiol 1995;16: 1347-52 Medline

21. Leach JL, Wolujewicz M, Strub WM. Partially recanalized chronic dural sinus thrombosis: findings on MR imaging, time-of-flight MR venography, and contrast-enhanced MR venography. AJNR Am J Neuroradiol 2007;28:782-89 Medline

22. Mas JL, Meder JF, Meary E, et al. Magnetic resonance imaging in lateral sinus hypoplasia and thrombosis. Stroke 1990;21:1350-56 Medline

23. Leach JL, Jones BV, Tomsick TA, et al. Normal appearance of arachnoid granulations on contrast-enhanced CT and MR of the brain: differentiation from dural sinus disease. AJNR Am J Neuroradiol 1996;17:1523-32 Medline

24. Ferro JM, Morgado C, Sousa R, et al. Interobserver agreement in the magnetic resonance location of cerebral vein and dural sinus thrombosis. Eur J Neurol 2007;14:353-56 CrossRef Medline

25. Idbaih A, Boukobza M, Crassard I, et al. MRI of clot in cerebral venous thrombosis: high diagnostic value of susceptibilityweighted images. Stroke 2006;37:991-95 CrossRef Medline 